\title{
COMPARACIÓN DE LOS EFECTOS DEL MASAJE Y EL ESTIRAMIENTO SOBRE EL RENDIMIENTO
}

\author{
Adriana Alvarado y Walter Salazar \\ Escuela de Educación Física y Deportes \\ Universidad de Costa Rica, San José, Costa Rica \\ E-mail: adriana_alvarado@hotmail.com
}

\begin{abstract}
Resumen
Alvarado, A. y Salazar, W. (2003). Comparación de los efectos del masaje y el estiramiento sobre el rendimiento. Revista de Ciencias del Ejercicio y la Salud 3(1), 43-53. El propósito de este estudio fue determinar si las técnicas del masaje y el estiramiento activo influyen en el rendimiento físico. Participaron voluntariamente 28 sujetos físicamente activos (14 hombres y 14 mujeres), con edades comprendidas entre 18 y 37 años, los cuales realizaron tres pruebas de rendimiento físico: el salto Sargent, la prueba de velocidad de 30 m y una prueba sub-máxima de fuerza para extensión de rodilla. Para la realización de estas pruebas se designaron seis diferentes secuencias de las mismas, que luego se aleatorizaron a los participantes. Los sujetos se debían presentar una vez a la semana durante dos semanas de forma tal que participaran en ambos tratamientos, después de realizar un calentamiento de 10 minutos de trote, los sujetos procedieron a realizar el pretest en la secuencia que aleatoriamente se les asignó, posterior a esto se realizaron los tratamientos: un tratamiento consistía en un masaje tipo effleurage de 20 minutos en total en ambos miembros inferiores (10 minutos, en cada pierna, 5 minutos prono y 5 minutos supino) y el tratamiento de estiramiento consistía de un estiramiento estático dirigido de 10 minutos tanto para miembros inferiores como para tronco. Una vez finalizado los tratamientos los sujetos realizaban el post test, en la misma secuencia de pruebas del pretest.: Para el análisis de los datos se realizaron tres ANOVAs $2 \times 2 \times 2$ (mediciones $x$ tratamiento $x$ género) y de acuerdo con esto se encontró una interacción triple significativa en la prueba de velocidad $(p<0.05)$ y diferencias estadísticamente significativas con relación al sexo $(p<0.05)$ en las tres pruebas físicas. Los resultados obtenidos por este estudio muestran que no existe diferencia entre el realizar un estiramiento activo o recibir un masaje antes de realizar actividad física, es decir, pueden ser utilizados como forma de calentamiento previo al ejercicio o simplemente se puede prescindir de ellos, sin que éste llegue a afectar en forma negativa el rendimiento del practicante. PALABRAS CLAVES: masaje, estiramiento, rendimiento físico, fuerza, potencia, velocidad.
\end{abstract}

\section{INTRODUCCIÓN}

En la actualidad ya nada asombra al mundo con respecto a resultados deportivos. Récords que se mantuvieron intocables por años, se ven hoy rotos a menudo y barreras que el propio hombre consideró infranqueables para un ser humano se ven rebasadas con relativa facilidad. Estos cambios no serían posibles si a la hora de la planificación y de la preparación física y deportiva, no hubieran sido aplicados novedosos adelantos científicos y experiencias llevadas a cabo por múltiples disciplinas del género de actividad físico deportiva (Leyva y Castro, 2001)

El ejercicio físico y la ejecución del deporte se hacen posibles gracias a las fuerzas desarrolladas por la acción voluntaria de los músculos del cuerpo a través del sistema esquelético, es así como se puede definir la actividad física, como cualquier actividad que genere fuerza por la activación de este sistema (Knuttgen y Komi, 1993). Igualmente, Annicchioarico (2002, p 1), la define como "cualquier movimiento corporal intencional, realizado con los músculos esqueléticos, que resulta en un gasto de 
energía y en una experiencia personal que permite interactuar con los seres y el ambiente que lo rodea".

Autores como, Franks (1983) y Leyva y Castro (2001), sostienen que para toda actividad física, existe una serie de acciones previas (incremento de la excitabilidad y la labilidad de los centros nerviosos, facilitación de la excitación y la activación de los hábitos motores ya adquiridos, incremento de la velocidad de las reacciones bioquímicas, incremento de la actividad de los órganos cardiaco y respiratorio, aumento de la temperatura corporal lo que provoca una disminución de la viscosidad muscular, una disociación de la hemoglobina en los tejidos, etc.) que preparan al organismo del practicante de forma óptima, permitiéndole asimilar con mayor efectividad las cargas de trabajo; a esa actividad previa es lo que se conoce como calentamiento.

Según Franks (1983, pp. 341-342), existen tres tipos de calentamiento físico con relación a la ejecución o tarea que se ejecuta, los cuales son:

1. El idéntico a la ejecución, el cual consiste en un pre-juego, en el cual el atleta utiliza como calentamiento la técnica con la que normalmente se desempeña en el juego (misma velocidad, distancia, forma, etc.)

2. El directo, que consiste en la utilización de la técnica en forma separada, solo un porcentaje de ésta es la que se utiliza como calentamiento, por ejemplo lanzar un disco realizando el movimiento completo de hombro y brazo pero no la velocidad real.

3. El indirecto, que tiene como propósito influenciar cambios en el rendimiento por medio del aumento de la temperatura de la sangre en el músculo. Ejemplo de éstos sería, correr, realizar ejercicios de estiramiento o de ejercicios de fuerza, tomar un baño caliente o realizarse un masaje.

Ahora bien, entre los diferentes elementos de los cuales se compone una sesión de actividad físico-deportiva; el calentamiento, se encuentra en la parte preparatoria de la práctica, la cual cumple con dos objetivos específicos: elevar el nivel de capacidad para generar trabajo y preparar la actividad del sistema nervioso central y del aparato neuromuscular del practicante (Leyva y Castro, 2001).

Por consiguiente, basándose en la clasificación de los tipos de calentamiento que existen y siendo el calentamiento indirecto el más popularmente utilizado (Franks, 1983), se tomó para fines de este estudio como los tratamientos: el masaje y los ejercicios de estiramiento; ya que diferentes investigadores han reportado los efectos de ambos tratamientos en el ámbito fisiológico, músculo-esquelético, piel, circulatorio, linfático y psicológico.

\section{El masaje: definición, efectos y propósito.}

Se define como masaje, la manipulación de los tejidos blandos de forma manual (White, 1998; Vickers y Zollman, 1999 y Pino 2001).

Según Prentice (1990), Callaghan (1993), Andree-Yicz (1993), Caffarelli y Flint (1993) y Vickers, Zollman y Reinish (2001), entre las diferentes técnicas de masaje se pueden encontrar: el effleurage, el pétrissage, y la vibración.

- El effleurage o roce, puede ser superficial o profundo; donde el profundo se debe realizar siguiendo el curso venoso y linfático hacia el corazón y el superficial se debe realizar de forma centrípeta, lo que produce un desplazamiento de la piel en dirección a las fibras musculares de distal a proximal, por medio de deslizamientos suaves y rítmicos.

- La técnica pétrissage o amasamiento, que es un procedimiento más profundo en la que se tracciona y exprime la piel, el tejido subcutáneo y la masa muscular.

- La vibración que se utiliza en las extremidades para ayudar a disminuir el tono muscular.

El masaje tiene efectos científicamente estudiados como lo son: aumento de flujo 
sanguíneo, aumento de la recuperación muscular, aumento del transporte de las sustancias de deshecho, disminución del edema post trauma e influencia en la circulación linfática y aumento de la fuerza muscular en reposo pero no necesariamente en el músculo exhausto (Ljungfelt, Bornmyr, Lilja, Ravald y Westlin (1994); el aumento del ritmo cardíaco y la disminución de la presión sanguínea temporalmente (Ligtfoot, Char, McDermott y Goya, 1997); alivio de la tensión y los espasmos musculares (White, 1998); disminución de la tensión muscular, (Vickers y Zollman, 1999); aumento de la flexibilidad de los tendones y el tejido conectivo y aumento en el tiempo de recuperación en ejercicio intenso principalmente por el aumento de flujo sanguíneo (Tiidus y Shoemaker, 1995; Hemmings, Smith, Graydon y Dyson, 2000).

$\mathrm{Su}$ finalidad global es preparar al practicante para lograr el más alto nivel en el periodo de tiempo más breve y con el mínimo gasto de energía psicofísica, aumentando el nivel de desarrollo de las cualidades físicas, incrementando la capacidad de trabajo y perfeccionando el nivel de una modalidad deportiva concreta, por medio de una variedad de técnicas como el roce, el amasamiento, la fricción, la percusión y las sacudidas (Andree - Yicz, 1993).

\section{El estiramiento: definición, efectos y propósito.}

El estiramiento muscular, por su parte, se entiende como la amplitud de movimiento de las unidades músculo tendinosas, a lo largo de un periodo de tiempo de una sola articulación o a través de varias articulaciones (Prentice, 1999).

Según Shellock y Prentice, (1985); Etnyre y Lee, (1988); Brandy, Irion y Briggler (1997) y Handel, Horstmann, Dickhuth y Gülch, (1997), entre las diferentes técnicas de estiramiento se pueden encontrar:

- La extensión balística: que se basa en la repetición de movimientos de rebote del músculo agonista lo que produce extensiones rápidas del músculo antagonista, lo que se dice puede causar micro desgarros dentro de la unidad músculotendinosa.

- La extensión estática: que implica el estiramiento de un músculo hasta un punto de incomodidad para el músculo antagonista determinando, colocándolo en una posición de extensión máxima y después mantenerlo en esa posición durante un lapso de tiempo determinado.

- La facilitación neuromuscular propioceptiva: que implica contracciones y estiramientos alternativos.

El estiramiento posee como efectos científicamente estudiados: cambios en el rango de movilidad de las articulaciones y excitabilidad neuromuscular (Hutton, 1993 y Prentice, 1999); elongación del tejido blando, reducción del dolor muscular antes de ejercitarse, reducción del riesgo de lesiones y aumento en el rendimiento físico (Lund, Vestergaard-Poulsen, Kanstrup y Sejrsen, 1998 y Herbert y Gabriel, 2002).

$\mathrm{Su}$ objetivo principal es el de preparar los tendones y ligamentos para un futuro movimiento, lo que permite una mayor flexibilidad, una disminución de la tensión y la rigidez, un aumento de la coordinación y la agilidad y un incremento de la circulación muscular (Prentice, 1999).

Entonces bien, en lo que se refiere al masaje y tomando en cuenta una de sus características como lo es el aumento en el flujo sanguíneo, Ljunfelt et al, (1994), estudiaron el cambio de éste, en la pantorrilla de 12 sujetos de ambos sexos, con edades comprendidas entre 16 y 48 años, con el fin de determinar el efecto de los linimentos aplicados con o sin masaje en el cambio del flujo sanguíneo en músculo y la piel. Para ésta investigación se utilizaron dos diferentes linimentos (Frisco y Tigre Balm), que eran aplicados respectivamente uno en la pantorrilla derecha y otro en la pantorrilla izquierda, por medio de dos diferentes técnicas de aplicación (masaje pretissage / aplicación sin masaje), donde para ambas 
aplicaciones se utilizó un tiempo de 10 min. Como instrumento de medición se empleó un láser Doppler, el cual registró el cambio del flujo sanguíneo de la pantorrilla cada $5 \mathrm{~min}$. durante 1 hora.

En este estudio, los autores, concluyen que no se demostró la acción del masaje para las variables estudiadas, ya que el linimento Frisco posee la propiedad de enfriar y el linimento Tigre la propiedad de calentar y enfriar la parte del cuerpo donde es aplicado, por lo que los efectos del masaje no resultaron ser claros sobre el cambio en el flujo sanguíneo.

Así mismo, argumentan que los linimentos son usados por parte de los atletas con o sin masaje, como preparación para la competición pero que poco se ha estudiado acerca de los efectos en el flujo sanguíneo y el cambio en el rendimiento del músculo sobre el cual son aplicados.

Tiidus y Shoemaker (1995), estudiaron la percepción del dolor después del ejercicio, el cambio del flujo sanguíneo y la recuperación del músculo cuadriceps de una de las piernas de sus sujetos, luego de que éstos habían realizado trabajo excéntrico en ambas piernas; los investigadores descubrieron que inmediatamente después del ejercicio el torque isométrico y dinámico había descendido en aproximadamente un $70 \%$ en comparación a los valores medidos pre-ejercicio. Los autores (1995), se basaron en la teoría que el masaje acelera el tiempo de recuperación del músculo después del ejercicio intenso, por lo que realizaron masaje post-ejercicio en la primera sesión y ejecutaron tres sesiones de masaje, tres días posteriores al ejercicio encontrando que los valores pre-ejercicio para ambas piernas (masaje / control) tendieron a regresar muy lentamente a sus valores normales través de los 4 días de masaje. En éste estudio, los investigadores no encontraron diferencias en el torque isométrico ni dinámico entre la pierna masaje y la pierna control. Así mismo, el flujo sanguíneo que fue medido por un ultrasonido Doppler en la arteria femoral, no presentó diferencias sobre los niveles de descanso, y para finalizar, el nivel de percepción del dolor sí tendió a disminuir en la pierna que recibió masaje de 48 a 96 horas post-ejercicio.

Por lo que se concluye que el masaje no es una modalidad de tratamiento efectivo para la disminución de los largos periodos de recuperación post-ejercicio, por cuanto, su uso para este propósito debe de ser cuestionado.

Sin embargo, Tiidus (1997), concluye en su revisión que existe poca evidencia científica de un impacto significativo en la disminución del tiempo de recuperación o de los factores psicológicos asociados con el proceso de recuperación por parte del masaje. Al mismo tiempo que recalca cuatro circunstancias en las cuales el masaje puede afectar, cuando se aplica en el músculo sobre entrenado como por ejemplo: 1) daño muscular causado por una acción muscular excéntrica, 2) retención y recuperación de la fuerza muscular y el rendimiento en un músculo dañado por una fuerza excéntricomecánica, 3) reducción o retraso del dolor en un músculo dañado por una fuerza excéntrico-mecánica, 4) recuperación de la fuerza muscular y el rendimiento después de realizar ejercicio anaeróbico. Lo que indica que para estos cuatro factores los efectos del masaje no serán funcionales, si no que pueden causar un deterioro muscular mayor al causado por el ejercicio intenso.

Con relación al rendimiento, Rinder y Sutherland (1995), estudiaron el rendimiento del músculo cuadriceps trabajando con 20 sujetos saludables de ambos sexos. Los participantes se aleatorizaron a dos tratamientos (masaje / descanso), en donde cada sujeto participó en dos sesiones experimentales; la primera sesión experimental consistía en realizar el máximo de extensiones de rodilla en ambas piernas hasta la fatiga, seguidamente se les realizaba el tratamiento de $6 \mathrm{~min}$. de masaje en una de las pierna o simplemente descansaban durante 6 min. terminado ese lapso de tiempo, los sujetos debían realizar nuevamente las extensiones de rodilla hasta la fatiga, una 
semana después cada sujeto debía realizar la segunda sesión experimental donde se repetía el proceso pero con la condición de tratamiento alternada.

Estos autores encontraron que después de 6 minutos de masaje ocurría un aumento significativo en el número de repeticiones que podía realizar el músculo cuadriceps analizado en comparación al músculo que se mantuvo en descanso (pierna control).

Así mismo, Hemmings et al, (2000), investigaron el efecto del masaje en el ejercicio repetitivo, el rendimiento, la eliminación del lactato y la percepción de recuperación después de recibido el masaje, por medio de la medición del lactato sanguíneo, la concentración de glucosa, la frecuencia cardiaca y la percepción del dolor.

Para esta investigación se trabajó con 8 boxeadores aficionados, con una edad promedio de 24 años, los cuales se aleatorizaron al siguiente protocolo: un calentamiento de $10 \mathrm{~min}$., seguidamente, debían golpear un ergómetro de boxeo durante un tiempo de 5.2 minutos simulando una pelea con un minuto de descanso entre rounds (rendimiento 1); luego recibían uno de dos tratamientos (combinación de masaje effleurage y petrissage de 20 minutos en total o un descanso de 35 minutos que sirvió como control). Por último, debían volver a golpear el ergómetro otros 5 rounds (rendimiento 2). Una semana después realizaban de nuevo el mismo protocolo, pero con la condición de tratamiento alternada.

Los investigadores no encontraron diferencias significativas entre los tratamientos (masaje / descanso), a pesar de mostrarse un detrimento en la fuerza del golpe entre el rendimiento 1 y rendimiento 2, pero sí se mostró estadísticamente que la intervención del masaje aumenta significativamente la percepción de recuperación en comparación al grupo de descanso. Con relación a la eliminación del lactato, el estudio encontró que el masaje no tiene efecto en la eliminación de éste, en comparación a una condición de descanso.
En conclusión, los investigadores ratifican que el masaje no tiene efecto en los deportes repetitivos, pero que a su vez, la utilización de éste no causa el detrimento en la ejecución física, por el contrario insisten en que el beneficio físico después de realizar ejercicio debe ser examinado en conjunto con la restauración psicológica.

En relación al estiramiento, High y Howley (1989) estudiaron el efecto del estiramiento estático y el calentamiento asociado a los niveles de dolor producido en el músculo cuadriceps por medio de la prueba Step (que consiste en realizar un trabajo concéntrico con pierna derecha y un trabajo excéntrico con la pierna izquierda hasta el agotamiento).

Esta investigación contó con 62 sujetos de ambos sexos, los cuales se asignaban aleatoriamente a 4 grupos: 1) realizaba estiramiento estático y la prueba Step; 2) realizaba caminata como calentamiento y la prueba Step; 3) una combinación de estiramiento estático, caminata y la prueba Step y 4) solamente realizó la prueba Step.

Se encontró, que había cierta diferencia entre el trabajo excéntrico y concéntrico ya que el excéntrico producía mayor dolor, pero al final se concluye que el estiramiento y el calentamiento no previenen el dolor del ejercicio exhaustivo.

Igualmente, Lund et al, (1998), estudiaron la relación entre la técnica de estiramiento pasivo y el dolor post-ejercicio en el músculo cuadriceps de 7 mujeres físicamente activas, por medio de la medición de fosfocreatina, creatina quinasa (CK) y la escala visual análoga durante un tiempo aproximado de una semana, en la cual se medían los sujetos una vez al día.

El trabajo del músculo cuadriceps consistía en realizar extensiones de rodilla de forma excéntrica hasta el agotamiento y el tratamiento de estiramiento consistía en realizarles estiramiento pasivo en la pierna tratada durante $30 \mathrm{~s}$. con intervalos de tiempo de 30, 75 y 95 minutos después de realizado el ejercicio. Se concluyó, que al no existir 
diferencias significativas el estiramiento pasivo no tiene influencia sobre el aumento en la CK en el plasma, la aparición de dolor muscular, la disminución de la fuerza y la disminución de fosfocreatina.

Por otra parte, en los estudios que combinan ambas técnicas; Rodenburg, Steenbeek, Schieereck y Bar (1994), estudiaron el efecto de la combinación de los ejercicios de estiramiento, el calentamiento y el masaje en el dolor producido por el ejercicio excéntrico. Para ello, midieron la CK y la mioglobina.

En este estudio se utilizaron 50 sujetos de ambos sexos, los cuales realizaban durante 30 minutos ejercicios excéntricos de bíceps y quienes se aleatorizaron a dos grupos a) experimental: calentamiento y estiramiento pre-ejercicio y masaje post-ejercicio y $b$ ) control: calentamiento y estiramiento preejercicio). Además, el dolor se midió a la hora, a las 24 horas, a las 48, 72 y 96 horas post-ejercicio.

Aunque, no encontraron diferencias entre grupos, sí hubo una tendencia a que la combinación de los tres tratamientos produjeran una reducción en los efectos negativos del ejercicio excéntrico.

De manera similar, Ligtfoot et al, (1997), investigaron la aparición del dolor post-ejercicio y a la vez determinaron si el masaje alteraba la respuesta inflamatoria que produce el ejercicio excéntrico; esto, mediante una escala visual análoga y la regla de Simpson. Para ello, trabajaron con 31 sujetos colegiales de ambos sexos, quienes debían realizar ejercicio excéntrico a nivel de ambas pantorrillas y que fueron divididos aleatoriamente en tres grupos a) grupo de estiramiento, $b$ ) masaje petrissage y $c$ ) el control), donde todos los tratamientos fueron de 10 min. y sólo se trato la pantorrilla izquierda.

En esta investigación (1997), se concluyó que no existías diferencias significativas entre el volumen de la pantorrilla tratada y la no tratada, igualmente el nivel de dolor entre una y otra, no fue significativo a las 24 horas ni 48 horas después de realizarse el masaje, por lo que se concluye que el masaje no previene la aparición del dolor.

Ciertamente, las investigaciones sobre masaje y estiramiento en función de la recuperación y el rendimiento son escasas y los resultados mostrados por éstas no son del todo concluyentes, ya que se enfocan más en la prevención del dolor, ya sea durante, inmediatamente después o varias horas después de realizado el ejercicio. Con certeza, todavía se requiere de más conocimiento, en relación con estos tratamientos sobre el rendimiento físico, debido a que diferentes estudios (Franks, 1983; High y Howley, 1989; Andree - Yicz, 1993; Lund et al, 1998; Hemmings, et al, 2000; Leyva $\mathrm{y}$ Castro, 2001; Herbert y Gabriel, 2002), indican los efectos que tanto el masaje, como el estiramiento pueden producir en el rendimiento; pero al mismo tiempo mencionan la falta de estudios científicos sobre el tema.

Por lo tanto, el propósito de este estudio fue comparar dos tipos de calentamiento indirecto, según la clasificación de Franks (1983), como lo son el masaje y los ejercicios de estiramiento activo para determinar su efecto en el rendimiento físico y al mismo tiempo determinar si sus efectos son susceptibles con relación al género, ya que no se halló evidencia científica sobre este aspecto en la literatura consultada; siendo este estudio relevante en la medida que examina los resultados de ambas técnicas de forma pura.

\section{METODOLOGÍA}

\section{Participantes}

En este estudio participaron voluntariamente 28 sujetos (14 hombres y 14 mujeres) físicamente activos (realizaban ejercicio al menos tres veces a la semana), con edades comprendidas entre 17 y 47 años, para un promedio de $21,10 \pm 6,39$ años. 


\section{Instrumentos de medición}

Se utilizaron tres pruebas de rendimiento físico:

1. El salto Sargent, ya que es un buen estimador de la potencia del tren inferior y su confiabilidad y objetividad es de 0.93 como lo reporta Aragón (1996). Operacionalmente se tomó como la diferencia del alcance máximo del sujeto, con su mano extendida desde una posición estática en el mayor de tres saltos ejecutados desde la misma posición.

2. Una prueba sub-máxima de fuerza para extensores de rodilla, equivalente a un $90 \%$ de la fuerza máxima del tren inferior la cual posee una confiabilidad de $\mathrm{r}=$ 0.60 a 0.80 , según Knutzen, Brilla y Caine (1999). Esta prueba se basa en realizar $5 \mathrm{RM}$ y descansar 1 minuto, mientras se aumenta la carga gradualmente hasta que el sujeto ya no pueda realizar más levantamientos.

3. La carrera de 30 metros no lanzados para medir velocidad, la cual posee una validez lógica y una confiabilidad de 0.95 (Hernández, 2000).

\section{Procedimientos}

Una vez explicado el propósito del estudio, se citó a los 28 sujetos para realizar las pruebas físicas, en dos diferentes horarios, el primer grupo de 14 sujetos trabajaba de 1 a 3 p.m. y el segundo grupo trabajaba de 3 a 5 p.m..

Antes de iniciar las pruebas se aleatorizaron los 28 participantes a seis diferentes grupos, en los cuales se variaba la secuencia de las pruebas a realizar, (donde $\mathrm{V}$ es (velocidad), P (potencia) y F (fuerza)) por ejemplo:

Grupo 1: V, P, F Grupo 2: F, P, V Grupo 3: P, V, F Grupo 4: V, F, P Grupo 5: P, F, V Grupo 6: F, V, P
Así, cada sujeto participó en dos sesiones experimentales en las cuales debían realizar tres pruebas físicas en un orden que previamente se les había otorgado y el tratamiento que aleatoriamente se realizó en esa sesión (en este caso el tratamiento podía ser el masaje o la realización de ejercicios de estiramiento).

Asimismo, el estudio se realizó en cinco pasos, tanto para mantener el orden del mismo, como para evitar contratiempos a la hora de realizar la medición de las diferentes pruebas, ya que cada prueba y tratamiento tenía un tiempo exacto de duración, de esta manera se utilizaba un estándar en el tiempo para todos los participantes.

I paso: Se le explicaba al grupo de participantes las pruebas físicas que debían realizar, la forma correcta de saltar, el lugar donde debían correr y la posición inicial para lograr el levantamiento de peso adecuadamente.

II paso: Consistía en realizar el calentamiento inicial el cual los participantes debían trotar durante 10 minutos.

III paso: Finalizado el calentamiento, se realizaban las primeras mediciones de las pruebas físicas ( $\mathrm{F}, \mathrm{P}, \mathrm{V}$, etc.)

IV paso: Se realizaba alguno de los dos tratamientos:

\section{Masaje:}

Para el tratamiento de masaje se utilizó la técnica de masaje effleurage recomendado Hoffa, Gocht y Storck (1985) y Tiidus y Shoemaker (1995), pues es un tipo de masaje en el que se realizan movimientos de la periferia hacia el centro, siguiendo la dirección de las fibras musculares desde su inserción hasta su origen realizando deslizamientos leves, suaves y rítmicos.

El masaje se realizó en ambas piernas, 10 minutos en cada pierna $(5 \mathrm{~min}$. en prono y $5 \mathrm{~min}$. en supino) desde el tobillo hasta el muslo sin tocar las articulaciones de 
tobillo y rodilla para contabilizar un total de 20 minutos, con base en lo recomendado por Andree - Yicz (1993).

\section{Ejercicios de estiramiento}

Para el tratamiento de estiramiento se utilizó una rutina de estiramiento estático de forma activa dirigida, para tren inferior tomando en cuenta los músculos que intervenían en las pruebas físicas que se estaban realizando (cuadriceps, isquiotibiales, aductores, abductores, psoas ilíaco, sóleos, gemelos, peroneos y tibiales). El tiempo total de estiramiento fue de 10 minutos.

V paso: Se realizaba la segunda medición de las pruebas físicas, en el mismo orden de la primera medición.

\section{Análisis Estadístico}

El análisis de los datos se realizó con ayuda del programa SPSS 8.0 para Windows, obteniéndose de esta manera la estadística descriptiva y la estadística inferencial. Para esto se realizaron tres ANOVAs de medidas repetidas $(2 \times 12) \times \quad$ (tratamiento $\mathrm{x}$ mediciones) $\mathrm{x}$ género. Finalmente se determinó la proporción de varianza explicada por medio del cálculo estadístico de omega al cuadrado $\left(\omega^{2}\right)$.

\section{RESULTADOS}

A continuación, en la Tabla 1, se presenta la estadística descriptiva correspondiente a los tratamientos en las dos mediciones para cada género según las tres variables dependientes (potencia, fuerza, y velocidad).

Tabla 1.

Estadística descriptiva correspondiente a las variables dependientes según el tratamiento las mediciones y el género.

\begin{tabular}{cccccc}
\hline & & \multicolumn{2}{c}{ Mujer } & \multicolumn{2}{c}{ Hombre } \\
\cline { 3 - 6 } Variable & Tratamientos & Pretest & Postest & Pretest & Postest \\
\hline Potencia & Masaje & $32.42 \pm 7.62$ & $32.78 \pm 5.19$ & $50.14 \pm 8.86$ & $52.5 \pm 5.78$ \\
$(\mathbf{c m})$ & Estiramiento & $32.78 \pm 5.19$ & $32 \pm 6.93$ & $53.71 \pm 6.48$ & $51.42 \pm 6.75$ \\
\hline Fuerza & Masaje & $9.35 \pm 2.27$ & $9.42 \pm 2.37$ & $16.64 \pm 4.89$ & $16.64 \pm 4.89$ \\
$(\mathbf{k g})$ & Estiramiento & $8.85 \pm 2.10$ & $9.28 \pm 2.26$ & $16.64 \pm 4.89$ & $17.42 \pm 4.27$ \\
\hline Velocidad & Masaje & $6.33 \pm 0.87$ & $6.78 \pm 0.99$ & $5.14 \pm 0.30$ & $5.17 \pm 0.45$ \\
$(\mathbf{s})$ & Estiramiento & $6.61 \pm 0.57$ & $6.45 \pm 0.73$ & $5.17 \pm 0.45$ & $5.34 \pm 0.53$ \\
\hline
\end{tabular}

En la variable género, se encontraron diferencias estadísticamente significativas en las tres pruebas de rendimiento físico ( $\mathrm{p}<$ $0.05)$, para la prueba de fuerza $(\mathrm{F}: 33,59 \mathrm{y}$ un $\omega^{2}=50,35 \%$ ), para la prueba de potencia (F: 91,05 y un $\omega^{2}=65,93 \%$ ) y para la prueba de velocidad (F: 67,52 y un $\omega^{2}=51,79 \%$ ), lo que se observa como una misma tendencia, donde los hombres superan a las mujeres en las tres pruebas físicas; además, se encontró una interacción triple significativa en la prueba de velocidad, sin embargo ésta no fue analizada debido a que su índice de varianza explicada era menor al $1 \%\left(\omega^{2}=0.88\right)$, lo que muestra la anulación de este efecto por parte de la interacción significativa de los efectos principales (Keppel, 1982).

Aparte de estos datos no se encontraron diferencias estadísticamente significativas entre los diferentes tratamientos en ninguna de las pruebas físicas así como diferencias entre el pretest y el post test, luego de realizado el tratamiento (Ver Gráfico $\mathrm{N}^{\circ} 1$ ). 


\section{Gráfico 1.}

\section{Gráfico de cada una de las pruebas físicas según las mediciones y el sexo.}

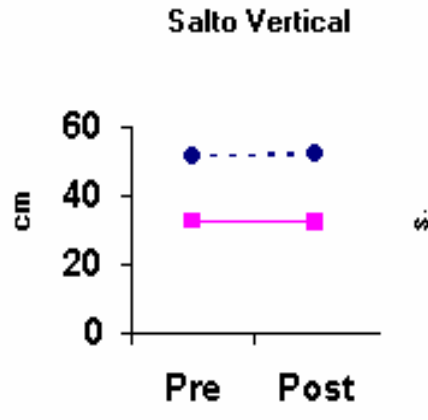

Salto Vertical

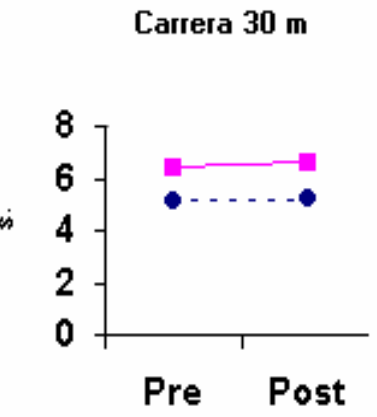

Mediciones
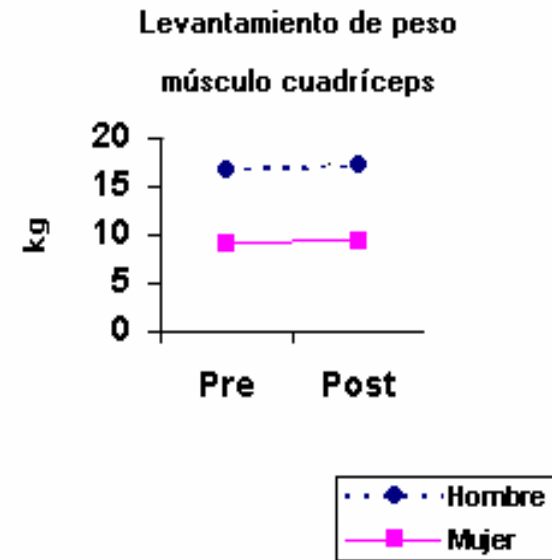

\section{DISCUSIÓN}

Esta investigación tenía como objetivo determinar si las técnicas de calentamiento indirecto (Franks, 1983) utilizadas antes de realizar la actividad física, influían en el rendimiento físico.

Los resultados presentados en este estudio están basados concretamente en variables que miden el rendimiento físico y que están involucradas en gran cantidad de deportes; particularmente los deportes individuales que demandan fuerzas extremas, coordinación, velocidad y potencia, los cuales son fácilmente cuantificables y pueden ser evaluados de forma objetiva, (Brooks, Fahey y White, 1996). Este tipo de pruebas físicas pertenecen a las actividades de tipo anaeróbico aláctico, en donde ocurre la liberación de la máxima potencia muscular, en un esfuerzo de intensidad muy elevada por un breve periodo de tiempo (de 20 a $120 \mathrm{~s}$ de duración) y en donde se incluye tanto el trabajo dinámico como el estático con la participación de grandes grupos de masas musculares (Mc Ardle, Katch y Katch, 1990; López y Legido, 1991).

Cabe aclarar que todos los artículos mencionados en esta investigación, hacen hincapié en la gran carencia de estudios concretos con respecto al tema de rendimiento y en especial a la especificación que los efectos que ambos tratamientos producen sobre éste.

La utilización de acciones previas para preparar el organismo, antes de realizar actividad física, son acogidas como elemento importante del entrenamiento físico y mental por parte de entrenadores y atletas, quienes ven en éstos una práctica útil, las cuales son cuestionadas en poca medida, debido a la gran popularidad de la que han formado parte tanto en el deporte en general como en la medicina deportiva (McArdle et al, 1990; Brooks et al, 1996; Leyva y Castro, 2001).

"Si bien es cierto, que el ámbito deportivo se abre cada vez más hacia el conocimiento probado científicamente, todavía hay quienes siguen absorbiendo información teórica y llenado inquietudes por medio de conocimientos establecidos previamente, lo que hace cuestionar hasta qué punto la teoría está adaptada a las condiciones reales que se viven en la practica" (Hernández, 2000, p. 34)

Autores, como, Andree - Yicz (1993); Ljungfeld et al, (1994); White (1998); Vickers y Zollman (1999) y Hemmings et al, (2000), sugieren que el masaje como tratamiento presenta evidencia contundente 
en relación con el beneficio psicológico más que el beneficio fisiológico en la fase de preparación y recuperación muscular, ya que como ellos mismos afirman, el masaje aumenta la relajación, disminuye la ansiedad, mejora la capacidad de sueño, aumenta el nivel de endorfinas y por ende la sensación de bienestar, por lo cual, estos beneficios no deben ser subestimados. Contrariamente a esto, Ligtfoot et al, (1997) refutan los beneficios psicológicos del masaje aseverando que éste por sí solo no puede producir cambios en el carácter ni el humor del practicante.

White (1998) menciona que el masaje se incluyó oficialmente como técnica médica terapéutica en las Olimpiadas de Atlanta en 1996 y es fundamental resaltar que, a pesar de que los beneficios fisiológicos como el aumento en el rendimiento, el alivio del dolor músculo esquelético, el aumento de movilidad articular y la capacidad de disminuir las lesiones, están comprobados con datos científicos significativamente concluyentes.

Por otra parte, en el presente estudio el efecto de los tratamientos no vislumbra cambios a nivel del rendimiento con relación al género, por lo que indistintamente del tratamiento que se realice, la capacidad física que tenga el practicante para realizar ejercicio no se ve afectada de una ni de otra forma.

En conclusión, los resultados obtenidos por este estudio muestran que no existe efecto significativo en el rendimiento al realizar un estiramiento activo o recibir un masaje antes de realizar ejercicio físico, ya que al no encontrarse una tendencia en el cambio de los tratamientos con relación al pre test $\mathrm{y}$ al post test, se puede señalar que tanto el masaje como el estiramiento no mejoran el rendimiento físico del practicante, pero tampoco causan un deterioro del mismo, resultante que es respaldada por Hemmings et al, (2002); es decir, se pueden seguir utilizando como forma de calentamiento previo al ejercicio o puede prescindirse de ellos antes de realizar actividad física.

\section{Agradecimientos}

A los colegas fisioterapeutas: Ana Morales, Mónica Sanabria, Silvia Abarca, Bernardo Durán, Carolina Miranda y María Emilia Venegas, ya que sin su ayuda no hubiera sido posible la realización de este estudio.

\section{REFERENCIAS}

Andree-Yicz, A. (1993). El masaje deportivo. (pp.182189). España: Taidotribo.

Aragón, L. (1996). Comparación de cuatro métodos para la medición del salto vertical. Revista Educación, 20 (1), 33- 40.

Brandy, W., Irion, J., y Briggler, M. (1997). The effect of static stretching on flexibility of the hamstring muscle. Physical Therapy, 77, 1090-1099.

Brooks, G., Fahey, T y White, T. (1996). Exercise physiology. ( $2^{\text {nd }}$ Edition). USA.: Human Bioenergetics and its applications.

Cafarelli, E., y Flint, F. (1993). The role of massage in preparation for and recovery from exercise. Physiotherapy in Sport, 16, 17-20.

Callaghan, M. (1993). The role of massage in the management of the athlete: a review. British Journal of Sports Medicine, 27, 28-33.

Etnyre, B., y Lee, E. (1988). Chronic and acute flexibility of men and women using 3 different stretching techniques. Research Quarterly for Exercise and Sport, 59, 222-228.

Franks, D. (1983). Ergogenics aids in sport. En Melvin Williams (pp. 340- 371). USA.: Human Kinetics.

Handel, M., Horstmann, T., Dickhuth, H., y Gülch, R. (1997). Effects of contract-relax stretching training on muscle performance in athletes. European Journal of Applied Physiology and Occupational Physiology, 76, 400-408.

Hemmings, B., Smith, M., Graydon, J y Dyson, R. (2000). Effects of massage on physiological restoration, perceived recovery, and repeated sports performance. British Journal of Sports Medicine, 34, 109 - 115.

Herbert, R y Gabriel, M. (2002). Effects of stretching before and after exercising on muscle soreness and risk of injury systematic review. BMJ, 325468.

Hernández, J. (2000). Efecto de tres tipos de entrenamiento para tren inferior: una verificación del principio de especificidad. Tesis de grado para optar por el grado de Magister Scientiae en Ciencias del Movimiento Humano. Universidad de Costa Rica. San José, Costa Rica.

High, D. y Howley, E. (1989). The effects of static stretching and warm- up on prevention of delayed - onset muscle soreness. Research Quarterly for Exercise and Sport, 60 (4), 357-363. 
Hoffa, Gocht, Storck- L. (1985). Técnicas del masaje. España: Jims.

Hutton, R. (1993). Strength and Power in Sport. (pp. 29-37). ( $3^{\text {rd }}$ Edition) Germany.: In Blackwell Scientific Publications.

Keppel, G. (1982). The Mixed Two-Factor Design: Simple Effects and Interaction Comparisons. In Design and Analysis a Researcher's Handbook (pp. 408-450) . (2 ${ }^{\text {nd }}$ Ed.) . Englewood Cliffs, New Jersey, USA.: Prentice-Hall, Inc.

Knuttgen, H y Komi, P. (1993). Strength and Power in Sport. (pp 3-4). (III edition). Germany: Blackwell Scientific Publications.

Knutzen, K. Brilla, L y Caine, D. (1999). Validity of 1RM prediction for older adults. Journal of Strength \& Conditioning Research, 13 (3), 242 246.

Ligtfoot, J., Char, D., McDermott, J y Goya, C. (1997). Immediate post exercise massage does not attenuate delayed onset muscle soreness. Journal of Strength \& Conditioning Research, 11 (2), 119-124.

Ljungfelt, C., Bornmyr, S., Lilja, B., Ravald, B y Westlin, N. (1994). The effects of liniment on blood flow. Scandinavian Journal of Medicine Science Sports, 4, 119-123.

López, J., y Legido, J. (1991). Umbral anaeróbico, bases fisiológicas y aplicación. España: Mc Graw Hill.

Lund, H., Vestergaard- Poulsen, P., Kanstrup, I y Sejrsen, P. (1998). The effect of passive stretching on delayed onset muscle soreness, and other detrimental effects following eccentric exercise. Scandinavian Journal of Medicine Science Sport, 8, 216-221.

McArdle, W. Katch, F. Katch, V. (1990). Fisiología del ejercicio. España: Alianza editorial Consejo Superior de Deportes.

Prentice, W. (1990). Modalidades terapéuticas en deportes. (II edición). USA: Times Mirror / Mosby.

Prentice, W. (1999). Técnicas de Rehabilitación. España: Editorial Paidotribo.

Rinder, A y Sutherland, C. (1995). An investigation of effects of massage on quadriceps performance after exercise fatigue. Complement Ther Nurs Midwifery, 1 (4), 99-102.

Rodenburg, J., Steenbeek, D., Schieereck, P y Bar, P. (1994). Warm - up, stretching and massage diminish harmful effects of eccentric exercise. International Journal of Sports Medicine, 15 (7), 414-419.

Sherlock F., y Prentice W. (1985). Warming- up and stretching for improved physical performance and prevention of sports related injures. Sport Medicine, 2 (4), and 267-278.

Tiidus, P y Shoemaker, J. (1995). Effleurage massage, muscle blood flow and long-term post-exercise strength recovery. Internal Journal of Sports Medicine, 16 (7), 478 - 483.
Tiidus, P. (1997). Manual massage and recovery of muscle function following exercise: a literature review. Journal of Orthopedic Sports Physical Therapy, 25 (2), 107 - 112.

Vickers, A y Zollman, C. (1999). Massage therapies. $B M J, 319,1254-1257$.

Vickers, A., Zollman, C., y Reinish, J. (2001). Massage therapies. West Journal Medicine, 175, 2002-204.

White, J. (1998). Alternative sports medicine. The Physician and Sports Medicine, 26, 92-105.

\section{Referencias tomadas de Internet (En Línea)}

Annicchiarico, R. (2002). La actividad física y su influencia en una vida saludable. Educación Física y Deportes: Revista Digital [En línea]. Disponible en: http:// www.efdeportes.com/efd51/saludt.htm.

Leyva, C y Castro, M. (2001). El calentamiento para la actividad físico- deportiva. Educación Física y Deportes, Revista Digital. [En línea]. Disponible en: http:// www.efdeportes.com/efd36/calent.htm.

Pino, J. (2001). Historia del origen y el desarrollo del masaje. Educación Física y Deportes: Revista Digital. [En línea]. Disponible en: http:// www.efdeportes.com/efd42/masaje.htm. 